\title{
Une mutation d'épissage peut dissocier retard mental et $\alpha$-thalassémie dans le syndrome ATR-X
}

La prédominance du retard mental chez les garçons est un fait établi dans toutes les séries étudiées, et les études familiales ont permis la description d'un nombre considérable de syndromes liés au chromosome X. En dehors du plus fréquent, lié à l'X fragile, la répartition n'en est pas aléatoire : les études de liaison ont en effet permis d'assigner à la région péricentromérique du bras long du chromosome X une quantité de formes cliniques variées, isolées ou syndromiques [1]. Un ou plusieurs gènes jouent-ils un rôle majeur ? Un syndrome non exceptionnel, décrit initialement par le groupe de DR Higgs (Oxford, GB), associant $\alpha$-thalassémie et retard mental (ATR-X), a fait l'objet d'une précédente nouvelle de médecine/sciences $\left(\mathrm{m} / \mathrm{s} n^{\circ} 6\right.$, vol. 11 , p. 910). Elle rapportait l'identification du gène responsable, dénommé $X H 2$ ou XNP, localisé en Xq13.3 [2]. La séquence de l'ADNc de ce gène a révélé l'analogie de son produit avec les protéines de la superfamille d'hélicases; il est intéressant de noter que les femmes transmettrices ont une inactivation de l'X extrêmement biaisée dans les tissus représentant le mésoderme (sang), l'endoderme (muqueuse buccale), l'ectoderme (racine des cheveux) indiquant le désavantage sélectif considérable du gène muté. Une nouvelle étape dans l'étude du syndrome ATR-X a été franchie, grâce à l'étude sur plusieurs années d'une grande famille française $[3,4]$. Dans cette famille de 45 personnes, trois individus sont affectés, deux cousins germains et un cousin éloigné. Chez les deux premiers, on retrouve l'ensemble du syndrome classique : retard mental profond, dysmorphies crânio-faciales, anoma- lies génitales et $\alpha$-thalassémie. Chez le cousin éloigné, en revanche, aucun signe d' $\alpha$-thalassémie, n'a été retrouvé malgré une recherche attentive d'inclusions d'hémoglobine $H$. L' $\alpha$ thalassémie étant décrite comme partie intégrante du syndrome ATR-X, la variabilité d'expression phénotypique au sein d'une même famille est surprenante et a déterminé les auteurs à une étude plus approfondie du gène $X N P$ [5]. Ce gène, en effet, est abondamment exprimé dans le cerveau, chez l'homme et chez la souris; l'expression est particulièrement élevée pendant l'embryogenèse et diminue à l'âge adulte. Mais il est exprimé aussi dans le placenta et, à un moindre degré, dans de nombreux tissus. La protéine, par ailleurs, contient plusieurs motifs, dont un tractus de 26 résidus glutamiques, susceptibles d'interactions variées. L'ensemble de ces caractères a fait émettre l'hypothèse d'actions pléiotropiques, et de la possibilité de phénotypes partageant des aspects communs mais d'autres aspects potentiellement variables.

Le gène $X N P$ est un grand gène, comportant 20 exons et s'étendant sur plus de $350 \mathrm{~kb}$. Les auteurs ont donc commencé par une étude par RTPCR des ARNm, isolés de lymphocytes périphériques ou de cultures de cellules lymphoblastiques. Une des paires d'amorces utilisées a montré un fragment de la taille attendue chez les membres témoins de la famille, un fragment plus court chez les deux cousins germains qui présentent un syndrome ATR-X typique, et la présence simultanée des fragments normal $(30 \%)$ et anormal chez le cousin éloigné ayant un retard mental sévère isolé. Chez les mères trans- mettrices, on n'a trouvé que le fragment le plus long, témoignant de l'extrême biais d'inactivation en faveur du chromosome X normal (>90\%). L'étude de l'ADN génomique dans la région impliquée n'a pas retrouvé la délétion observée au niveau des transcrits. Les jonctions exon/intron ont alors été explorées; une mutation $\mathrm{T} \rightarrow \mathrm{A}$, interrompant la séquence consensus polypyrimidique, a été trouvée en position -10 de la jonction 3', à l'état homozygote chez les trois malades, hétérozygote chez leurs mères transmettrices. La mutation, retrouvée sur ADN génomique, absente chez de nombreux témoins, semble donc responsable de l'excision d'un exon de $176 \mathrm{pb}$ (exon skipping). Le retentissement sur l'épissage de mutations du tractus polypyrimidique a déjà été décrit dans le cas de mutations de la $\beta$-globine et de la 21-hydroxylase [6,7].

Le phénomène original ici est l'hétérogénéité familiale pour une unique mutation. La distance familiale existant entre les cousins germains et le cousin éloigné peut cependant expliquer une différence de contexte génétique, qui agirait sur l'efficacité de l'épissage et, en conséquence, sur différents aspects du processus physiopathologique. Il faut noter aussi que l'épissage est variable selon les tissus, normal dans les précurseurs érythroblastiques chez l'un des malades, alors qu'il est anormal, cause d'une thalassémie chez les deux autres. En revanche, il est vraisemblablement constamment anormal dans le cerveau. Un phénomène très voisin au cours duquel une excision d'exon dépend de la longueur variable du tractus polypyrimidique a été observé dans le cas du gène CFTR 
[8] ; mais le phénomène est sans doute plus général et traduit la modulation polygénique de maladies monogéniques [9]. L'ensemble de l'observation montre que l'action du gène régulateur $X N P$, variable selon les tissus, permet de dissocier les différents aspects du phénotype. Son exploration reste donc justifiée chez des sujets présentant un retard mental sans $\alpha$-thalassémie, si sa liaison est établie avec la région Xq13. Il ne faut pas non plus négliger l'ensemble de la région Xq13-q21 qui pourrait contenir d'autres gènes responsables de retards mentaux liés à l'X.
1. Neri G, Chiurazzi P, Arena JF, Lubs HA. XLMR genes: update 1994. Am J Med Genet 1994; 51 : 542-9. 2. Gibbons RJ, Picketts DJ, Villard L, Higgs DR. Mutations in a putative global transcriptional regulator cause X-linked mental retardation with $\alpha$-thalassemia (ATR-X syndrome). Cell 1995; 80; 837-45.

3. Houdayer C, Toutain A, Ronce N, Lefort G, Sarda P, Taib J, Briault S, Lambert JC, Moraine C. $\mathrm{X}$-linked $\alpha$-thalassemia/mental retardation syndrome, linkage analysis in a new family further supports localization in proximal Xq. Ann Genet 1993; 36 : 194-9.

4. Lefort G, Taib J, Toutain A, Houdayer C, Moraine C, Humeau C, Sarda P. X-linked $\alpha$-thalassemia/mental retardation (ATR-X) syndrome. Ann Genet 1993 ; 36: 200-4.

5. Villard L, Toutain A. Lossi AM, Gecz J, Houdayer C, Moraine C, Fontès M. Splicing mutation in the ATR-X gene can lead to a dysmorphic mental retardation phenotype without $\alpha$-thalassemia.

D.L. Am J Hum Genet 1996; 58 : 499-505
6. Beldjord C, Lapouméroulie C, Pagnier J, Benabadji M, Krishnamoorthy R, Labie D, Bank A. A novel $\beta$-thalassemia gene with a single base mutation in the conserved polypyrimidine sequence at the 3' end of IVS 2. Nucleic Acids Res 1988; 16: 4927-35.

7. Higashi y, Tanae A, Inoue H, Hiromasa T, FujiiKuriyama Y. Aberrant splicing and missense mutations cause steroid 21-hydroxylase (P-450(C21)) deficiency in humans : possible gene conversion products. Proc Natl Acad Sci USA 1988; 85 : 748690.

8. Kiesewetter S, Macek M, Davis C, Curristin SM, Chu CS, Grahan C, Shrimpton AE, Cashman SM,

Tsui L, Mickle J, Amos J, Highsmith WE, Shuber A, Witt DR, Crystal RG, Cutting GR. A mutation in CFTR produces different phenotypes depending on chromosomal background. Nature Genet $1993 ; 5: 274-7$

9. Labie D, Elion J. Modulation polygénique des maladies monogéniques : l'exemple de la drépanocytose. médecine/sciences 1996; 12: 341-9. 